\title{
ON THE INTEGRATION OF A MICRODIALYSIS-BASED $\mu$ TAS WITH CALIBRATION FACILITY ON A SILICON-GLASS SANDWICH
}

\author{
Ad Sprenkels, Wouter Olthuis and Piet Bergveld \\ $\mathrm{MESA}^{+}$Research Institute, University of Twente, P.O. BOX 217, 7500 AE Enschede, \\ The Netherlands - Tel +31534893956,Fax+31534892287, Email a.j.sprenkels@el.utwente.nl
}

\begin{abstract}
In this paper the integration is discussed of all parts of a microdialysis-based micro Total Analysis System or $\mu$ TAS. In particular a microdialysis probe, a potentiometric and amperometric ion- and enzyme sensor and a calibration dosing pump have been developed separately using different precision machining techniques. By modifying and adapting these parts they can be realized in one generic technology consisting of a stack of a silicon and a glass wafer. The silicon wafer contains the double lumen microdialysis probe connections, a dosing pump chamber with meander formed cavities containing the calibration solutions and small cavities for both the potentiometric and amperometric sensor. The glass wafer contains all the electrical contacts and wires for the sensors, the pump and interconnections. Both wafers are anodically bonded to each other, yielding a hermetically sealed liquid handling system.
\end{abstract}

\section{INTRODUCTION}

Microdialysis has become a major standard sampling technique in clinical applications during the last 15 years. In this technique a double-lumen microdialysis probe is indwelled in the relevant organ tissue. Extracellular substances such as glucose and ions diffuse through the semi-permeable membrane into the perfusion liquid in the probe. This perfusion liquid is then pumped from the probe to appropriate analytical equipment to measure its composition outside the human body. In this way reliable measurements can be performed on clear samples without sensor fouling by particles such as cells and proteins. However, the connection between the actual microdialysis probe and the detector results in a large dead volume and long lag times, which is a serious drawback for real application in for instance intensive care units. By integrating the microdialysis probe, the sensors and a calibration facility on silicon, the size of the system is drastically reduced, resulting in a smaller sample volume, a reduced dead volume and short lag times. In this way measurements can be performed faster and more reliable than with conventional techniques. Such chips are called "lab-on-a-chip" or micro Total Analysis Systems or $\mu$ TAS. The MESA* Biosensor research group has started a project for the integration of all parts of a microdialysis-based $\mu \mathrm{TAS}$ on one chip. In particular the microdialysis probe, sensors and a calibration dosing pump have been developed separately using different precision machining techniques [1-4]. By modifying and adapting these parts they can be realized in one generic technology: a stack of a silicon wafer and a glass wafer that are anodically bonded.

Successively the various parts of a microdialysis-based $\mu$ TAS that have been developed until now will be discussed. Next the design of a complete microdialysisbased $\mu$ TAS that is realized in the generic silicon-glass technology will be shown. Finally some conclusions are given.

\section{COMPONENTS AND RESULTS}

In the first phase of the project a microdialysis probe has been realized in silicon [1], a potentiometric and an amperometric sensor in Perspex $[2,3]$ and an electrochemically actuated pump in a silicon-glass sandwich [4]. The aim now is to integrate all those parts on one chip using one generic technology: a silicon-glass bonded sandwich. The silicon wafer contains the double lumen tube connections, meander formed cavities which contain the calibration solutions with a dosing pump chamber, micro cavities for potentiometric and amperometric ion- and enzyme sensors. The glass wafer with a thin patterned Pt layer contains all the electrical contacts and wires for the sensors and pump. Both wafers are anodically bonded, yielding a hermetically sealed liquid handling system.

The following parts have been developed.

\section{A. Microdialysis probe}

The initially developed double lumen microdialysis probe connector consists of a sandwich construction of two $\mathrm{KOH}$ etched silicon wafers which, after bonding allow the easy insertion of two concentric fused silica capillaries, required to construct the probe. The connector forms an integral part of a double lumen type microdialysis probe and guides the flow of sample fluid ('dialysate') directly into a flow cell with space for integrated sensors (Fig. land Fig. 2). For the experimental evaluation of its performance a chloride selective sensor was integrated in the flow cell of the connector to continuously measure the chloride concentration in the dialysate flow. With this probe the induced lag time of the measurement has been decreased by

$3261^{\text {st }}$ Annual International IEEE-EMBS Special Topic Conference on Microtechnologies in Medicine \& Biology October 12-14, 2000, Lyon, France - 0-7803-6603-4/00/\$10.00@2000 IEEE 
a factor of more than 5 , as compared to a conventional probe connected to a flow through sensor [1]. Another benefit of the proposed direct coupling of double lumen microdialysis probes and microfluidic structures in silicon, is the fact that all critical fluidic connections, especially the probe/sensor connection, are kept inside, making the microanalysis system more rigid.

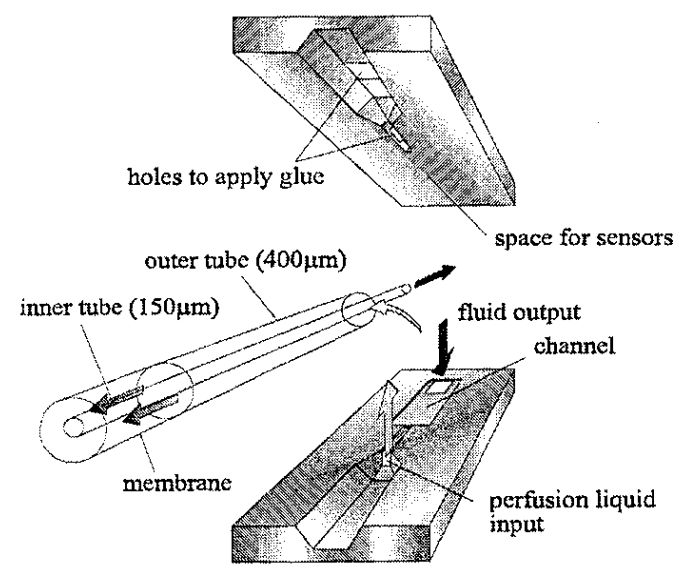

Fig. 1. Exploded view of the microdialysis probe/sensor construction.

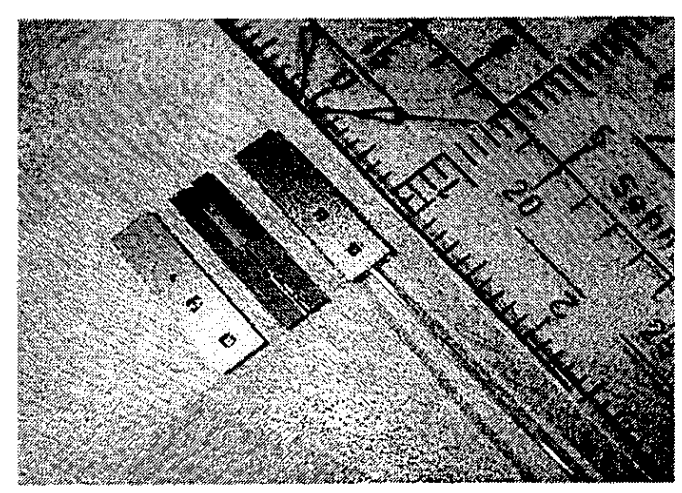

Fig. 2. Etched probe components (left) and assembled probe (right) next to a centimeter scale.

By adapting this design a comparable probe can be realized in a silicon-glass sandwich as shown in Fig. 3.

\section{B. Potentiometric sensor}

A flow-through potentiometric micro sensor which is based on semi-permeable tubing has initially been precision machined in Perspex (Fig. 3 and Fig. 4 ) in order to prove its operational principle [2]. Basically this ion selective sensor is of the liquid membrane type in which a dialysis tube carries the dialysate and is surrounded by an ion selective cocktail. Commercially available ionophores or ionexchanger cocktails are applied to provide ion selectivity. A number of flow-through micro sensors selective to different ions $\left(\mathrm{Na}^{+}, \mathrm{K}^{+}, \mathrm{Li}^{+}, \mathrm{pH}\right)$ were constructed and tested. Each showed good performance in a broad range of concentrations [2]. Advantages of the proposed approach are the generic technology to make sensors capable of measuring a wide variety of analytes for which cocktails are readily available and the fact that very small sub microliter amounts of sample can be measured. Secondly, in comparison with other sensors applied in micro channels, the impedance of the proposed flow-through sensor is much smaller because of the relatively large sensing surface formed by the tube wall.

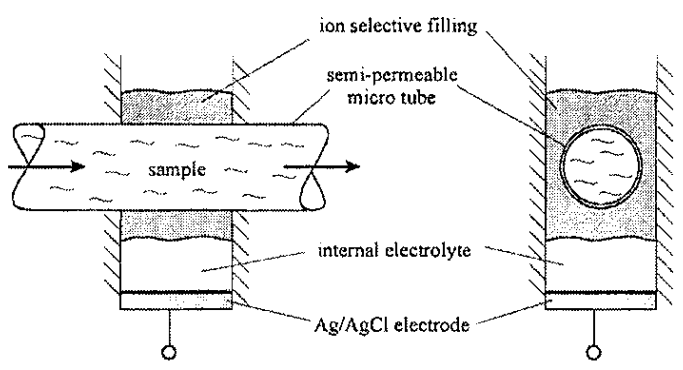

Fig. 3. Layout of the proposed flow-through potentiometric sensor based on semi-permeable tubing.

With some modifications this generic ion-selective potentiometric sensor can be realized in a silicon-glass sandwich as shown in Fig. 5 and Fig. 6.

After bonding the glass wafer on the silicon wafer the ionophore or ion-exchanger cocktail can be applied through the filling holes into the measuring cavity around the semipermeable tube. A Pt electrode is present on the glass wafer. On top of the $\mathrm{Pt}$ an $\mathrm{Ag} / \mathrm{AgCl}$ electrode is realized either by deposition of a $\mathrm{Ag}$ electrode and chlorination in a

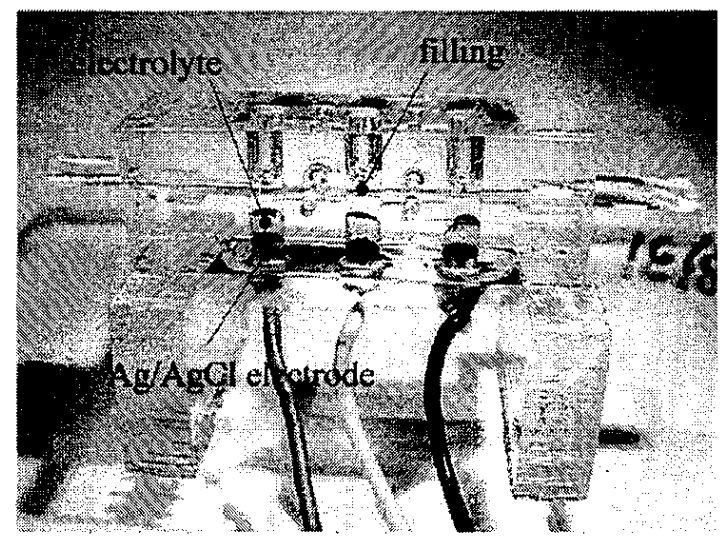

Fig. 4. Photograph of an array of three flow through sensors.

$0.1 \mathrm{M} \mathrm{HCl}$ solution, or by the application of a commercially available $\mathrm{Ag} / \mathrm{AgCl}$ paste. After application of a small 


\section{Poster 112}

amount of $\mathrm{KCl}$ solution the filling holes are closed with a small amount of epoxy.

Both glue holes alongside the sensor cavity are filled with a small amount of epoxy around the dialysis tube, fixing it and yielding a leakage-free liquid connection. In addition it serves as an electrical insulation between two adjacent sensors.

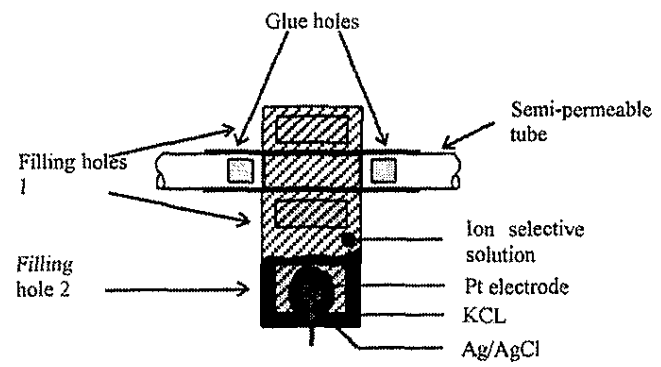

Fig. 5. Top view of the flow-through potentiometric sensor in asilicon-glass sandwich.

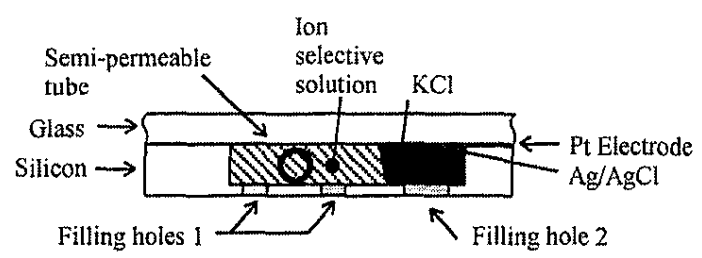

Fig. 6. Cross section of the silicon-glass potentiometric sensor.

\section{Amperometric sensor}

As is the case with the potentiometric sensors, also a generic flow-through amperometric micro enzyme sensor has been realized by precision machining in Perspex, which is based on semi-permeable dialysis tubing carrying the sample to be analyzed [3]. This tubing ( $300 \mu \mathrm{m}$ OD) is led through a small cavity, containing the working- and reference electrode as indicated in Fig. 7. By filling this cavity with a few microliter of an appropriate enzyme solution, an amperometric enzyme sensor results as shown in Fig. 8. As the dialysis tubing is impermeable for large molecular species such as enzymes, this approach does not require any immobilization chemistry, and as a consequence the enzyme is present in its natural free form. Based on this principle, amperometric sensors for lactate, glucose and glutamate were formed by filling cavities with buffered solutions containing respectively lactate-, glucose- and glutamate oxidase. All sensors showed a large linear range (0-35 mM for glucose, $0-3 \mathrm{mM}$ for lactate and $0.5 \mathrm{mM}$ for glutamate) covering the complete physiological range. The lower detection limit was in the order of $15-50 \mu \mathrm{M}$.

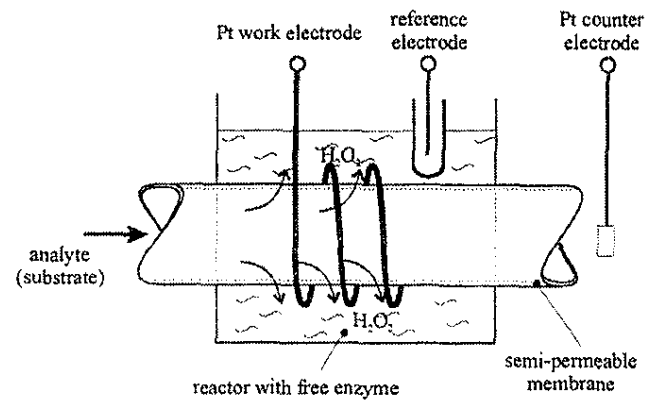

Fig. 7. Basic flow-through amperometric sensor geometry.

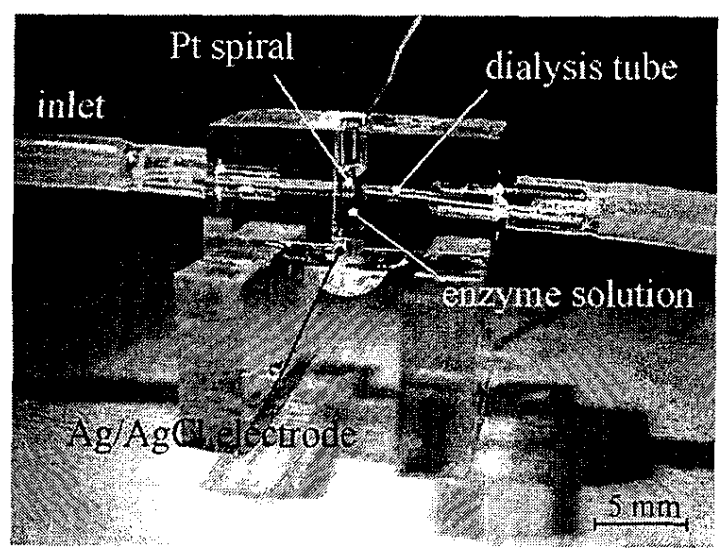

Fig. 8. Photograph of the amperometric flow-through sensor.

As shown in Fig. 9 and Fig. 10 this sensor principle can also be realized in a silicon-glass sandwich in a very similar layout as the aforementioned potentiometric sensor. For a proper operation of the amperometric sensor the necessary working electrode (WE) is present on the glass wafer adjacent to the dialysis tube. The required counter electrode is positioned in the liquid channel at some distance from the sensor itself.

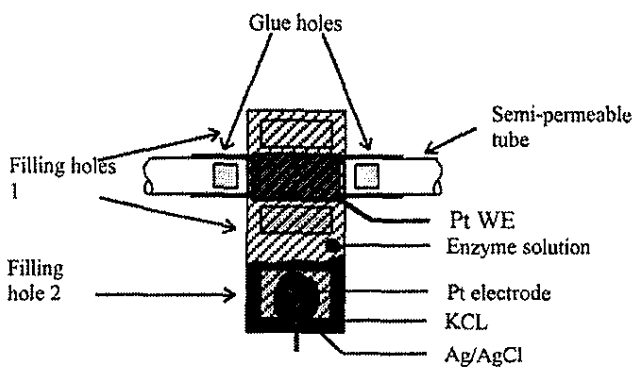

Fig. 9. Top view of the amperometric flow-through sensor in a silicon-glass sandwich. 


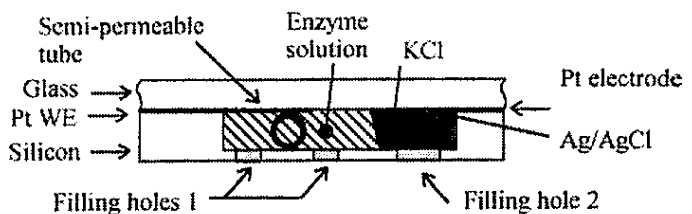

Fig. 10. Cross section of the silicon-glass amperometric sensor.

\section{Reference electrode}

As is evident from Fig. 11 the reference electrode is very similar to both aforementioned sensors. In fact it is equal to a $\mathrm{KCl}$ filled potentiometric sensor and will therefore not be discussed further.

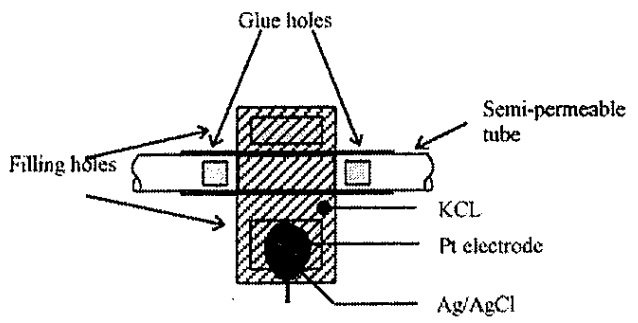

Fig. 11. Top view of an $\mathrm{Ag} / \mathrm{AgCl}$ reference electrode in a silicon-glass sandwich.

\section{E. Calibration-liquid pump}

A dual micromachined electrochemically actuated pump capable of dosing precise nanoliter amounts of liquid is shown in Fig. 12 [4]. The pump consists of a micromachined channel structure realized in silicon by reactive ion etching. On top of this structure a glass cover piece with $\mathrm{Pt}$ electrodes was bonded. The fluid to be dispensed is stored in a meander shaped reservoir "A", which is part of the channel structure. This meander starts in an electrolyte solution containing reservoir, on top of which two electrodes are positioned. By the electrochemical production of $\mathrm{H}_{2}$ and $\mathrm{O}_{2}$ gas bubbles via the electrolysis of water at electrodes 1,2 and 3 , liquid can be driven out of the meander. The measured volume displacements were in close agreement with theory. Pump rates as low as a few nanoliter per second could accurately be controlled via the actuation current through the electrodes. By continuously measuring the impedance between electrode 1 and 2 in the $\mathrm{H}_{2}$ generating chamber the total volume of dosed liquid can be monitored and controlled. By applying current pulses rather than a continuous current, preset amounts of fluid in the nanoliter range could be dosed successfully. Because this device has already been realized in a silicon-glass sandwich it can be incorporated without major modifications in the integrated complete $\mu$ TAS that will be discussed in the next chapter.

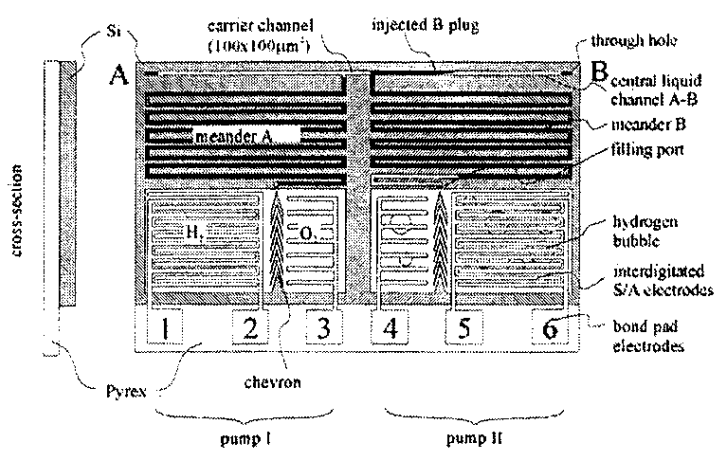

Fig. 12. Top view of the dual electrochemically actuated calibration pump.

\section{COMPLETE $\mu$ TAS DESIGN}

The lay out of the complete microdialysis-based $\mu$ TAS is shown in Fig. 13. It consists of a dual electrochemically actuated pump, an amperometric sensor (2), a potentiometric sensor (1), a reference electrode (RE) and a needle shaped microdialysis probe. In addition it contains a number of filling holes and glue holes. The filling holes are necessary to be able to fill the various parts of the system with the appropriate solutions. The glue holes are necessary to hermetically seal the various parts with appropriate epoxy.

In this design an external pump is used for delivering the perfusion liquid. As soon as an appropriate pump is developed, it will be incorporated into the system.

The total chip, excluding the probe, measures about $2.7 \times 1.5 \times 0.1 \mathrm{~cm}^{3}$. It consists of a silicon part containing the liquid channels, the filling and glue holes, and a glass part containing all the electrical wires for the sensors and pump. The actual probe consists of an inner fused-silica tube (150 $\mu \mathrm{m})$, through which, via the $\mathrm{IN}$ connection, a perfusion liquid is delivered by an external pump. The tip of the probe consists of a $300 \mu \mathrm{m}$ thick semi-permeable tube around the silica tube.

The outer tip of the probe is closed with a small drop of epoxy. The semi-permeable dialysis tube is connected inside the chip with a liquid channel that is connected with the calibration pump and the sensor-area. On top of this channel the counter electrode $\mathrm{CE}$ for the amperometric sensor is present.

The sensor-area consists of a second semi-permeable tube ending at the WASTE connection. It contains the potentiometric flow-through sensor (1), an amperometric flow-through sensor (2) and the reference electrode (RE). On the glass part 14 bonding pads are visible for the connection of the various components with appropriate measuring and controlling electronics. 


\section{Poster 112}

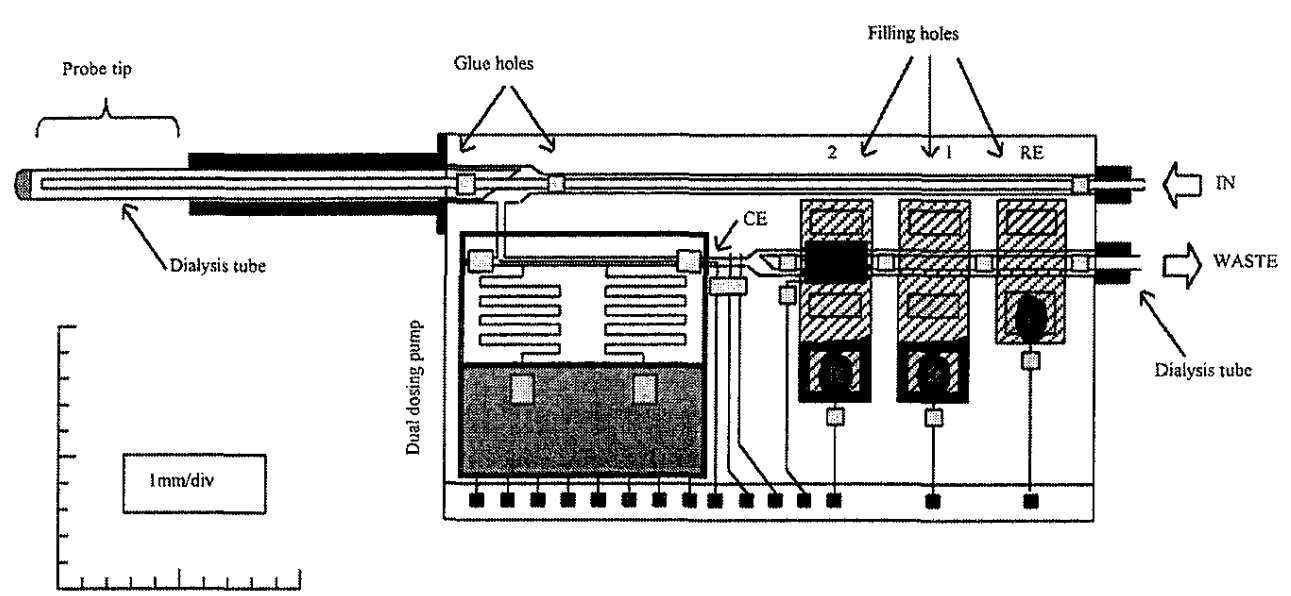

Fig. 13. Lay out of the complete $\mu$ TAS chip $\left(2.7 \times 1.5 \times 0.1 \mathrm{~cm}^{3}\right)$, including a microdialysis probe, two sensors, a reference electrode and a dual electrochemically actuated dosing pump for sensor calibration.

A complete measuring cycle starts with filling the probe tip with perfusion liquid through the $\mathrm{IN}$ connection by an external pump. Next the calibration pump delivers one or two small plugs of calibration liquid to the sensor area. During the next 1-3 minutes the perfusion liquid is enriched from its surroundings. At the same time the sensors are calibrated through the second semi-permeable tube crossing the sensor area.

After calibration of the sensors new perfusion liquid is pumped into the tip of the probe, pushing the enriched dialysate into the sensor area for analysis. During analysis the new perfusion liquid is enriched again from its surroundings and so on.

Depending on the stability of the sensors a second calibration step may be necessary after some time.

\section{CONCLUSIONS}

A $\mu$ TAS has been discussed comprising an inlet, a calibration facility with an electrochemically actuated pump, a potentiometric and an amperometric sensor and a reference electrode. The subsystems make use of one and the same technology: that of semi-permeable microdialysis membranes. The whole $\mu$ TAS can be realized in a two-layer sandwich of a silicon wafer, containing the fluidic channels and sensor cavities, and a glass wafer containing the electrical wiring. Advantages of this approach: due to the generic technology and the small size of the resulting system, a robust, reliable and fast responding analysis system is expected to be realized.

\section{ACKNOWLEDGMENT}

We would like to thank Mr. J. Bomer for the manufacturing of all devices and Dr. S. Böhm for his $\mathrm{PhD}$ research on the initial parts of the system. This work was financially supported by the Dutch Foundation for Fundamental Research on Matter (FOM).

\section{REFERENCES}

[1] Sebastian Bohm, Wouter Olthuis and Piet Bergveld, "A micromachined double lumen microdialysis probe for $\mu \mathrm{TAS}$ applications," The 13th European Conference on Solid-State Transducers (Eurosensors XIII), The Hague, The Netherlands, Sept. $1999,165-166$

[2] Sebastian Bohm, Wouter Olthuis, Piet Bergveld, "A generic design of a flow-through potentiometric sensor array," Mikrochim. Acta $134,237-243(2000)$.

[3] S. Bohm, D. Pijanowska, W. Othuis, and P. Bergveld, "A flowthrough amperometric sensor based on dialysis tubing and free enzyme reactors," The 14th European Conference on Solid-State Transducers (Eurosensors XIV), Copenhagen, Denmark, Aug. $2000,43-45$.

[4] S. Bohm, W. Olthuis, and P. Bergveld, "An integrated micromachined electrochemical pump and dosing system," J. Biom. Microdev. 1:2, 121-130, 1999. 